\title{
Study of Higgs Boson in Fermionic Decay Channels at CMS
}

\author{
Somnath Choudhury ${ }^{* \dagger}$ \\ DESY - Hamburg, Germany \\ E-mail: somnath. choudhury@cern.ch
}

\begin{abstract}
Results for the Standard Model Higgs Boson search are presented in the final states consisting of either two tau leptons or a bottom quark pair, based on proton-proton collision data collected in the years 2011 and 2012 at 7 and $8 \mathrm{TeV}$ respectively with the CMS experiment. Leptonic and hadronic decay channels for the tau lepton are included in the search. Different production mechanisms namely gluon fusion, vector boson fusion and associated production with vector bosons have been studied. The Standard Model Higgs boson search in the $b \bar{b}$ decay channel is studied in associated production with $\mathrm{W}$ and $\mathrm{Z}$ bosons. Interpretations of the data in the Minimal Supersymmetric Standard Model have also be given for both $\tau \tau$ and $b \bar{b}$ decay modes with the Higgs boson utilizing the final state for associated production with a b-jet to enhance the sensitivity of the minimal supersymmetric Higgs coupling to bottom quarks.
\end{abstract}

XXI International Workshop on Deep-Inelastic Scattering and Related Subject -DIS2013, 22-26 April 2013

Marseille,France

${ }^{*}$ Speaker.

${ }^{\dagger}$ for the CMS collaboration 


\section{Introduction}

The discovery of the mechanism of electro-weak symmetry breaking has been one of the primary goals of the Large Hadron Collider (LHC) at CERN. In the Standard Model (SM), electroweak symmetry breaking is achieved by the introduction of a complex scalar doublet leading to 4 physical degrees of freedom of which are the 3 "eaten" Goldstone bosons, namely the longitudinal states of the $\mathrm{W}^{+}, \mathrm{W}^{-}$and the $\mathrm{Z}$ boson, and the remaining one is a real physical scalar particle, which is the Higgs boson. The four main production mechanisms for Higgs boson in pp collisions are the gluon-gluon fusion mechanism having the largest cross-section, followed in turn by vector boson fusion (VBF), associated $\mathrm{WH}$ and $\mathrm{ZH}$ production, and production in association with top quarks. The relevant decay modes of the SM Higgs boson depend strongly on the decay branching fraction and its mass $m_{H}$. The Higgs boson discovered in the bosonic decay channels [1] around a mass of $125 \mathrm{GeV}$ makes the search in the fermionic decay channels $\tau \tau$ and $b \bar{b}$ very important and relevant as both these decay modes cover the low mass range. The results presented here are based on the two primary fermionic decay modes: $\mathrm{H} \rightarrow \tau \tau$ and $\mathrm{H} \rightarrow b \bar{b}$.

The Higgs boson suffers from quadratically divergent self-energy corrections at high energies. Numerous extensions to the SM have been proposed to address these divergences one of which is supersymmetry, a symmetry between fundamental bosons and fermions, which results in cancellation of the divergences. The Minimal Supersymmetric extension of the Standard Model (MSSM), requires the introduction of 2 Higgs doublets leading to 5 physical Higgs bosons, $\mathrm{h}$ and $\mathrm{H}$ being the CP-even scalar ones, $\mathrm{A}$ as the $\mathrm{CP}$-odd pseudoscalar boson and $\mathrm{H}^{+}$and $\mathrm{H}^{-}$are the charged ones. A search for MSSM Neutral Higgs Bosons in decays to a pair of tau leptons and bottom quarks has also been reported.

The analysis presented here is based on the 7 and $8 \mathrm{TeV}$ proton-proton collision data collected in the years 2011 and 2012 by the CMS detector [2] at the LHC. The detector sub-systems used for the analysis consists of a barrel assembly and two end-caps, comprising, in successive layers outwards from the collision region, the silicon pixel and strip tracker, the lead tungstate crystal electromagnetic calorimeter (ECAL), the brass/scintillator sampling hadron calorimeter (HCAL) enveloped in the superconducting solenoid, outside which are the gas-ionization chambers embedded in steel return yoke for the detection of muons.

\section{2. $\mathbf{H} \rightarrow \tau \tau$ decay mode}

Hadronically decaying $\tau$ leptons are reconstructed and identified using an algorithm called the Hadron Plus Strips (HPS) algorithm which is based on Particle Flow (PF) object reconstruction [3]. This technique identifies all stable individual particles produced in the event combining the capabilities of each sub-detector giving us the most precise measurement of the energy and direction of each particle and hence providing event description in the form of mutually exclusive particles. The HPS algorithm which starts with a PF jet for the hadronic tau reconstruction, targets the main decay modes by selecting particle candidates with one charged hadron and up to two neutral pions, or with three charged hadrons. A photon from a neutral pion decay can convert in the tracker material into an electron and a positron, which can then radiate bremsstrahlung photons. These particles give rise to several ECAL energy deposits at the same pseudorapidity $(\eta)$ value and separated in 

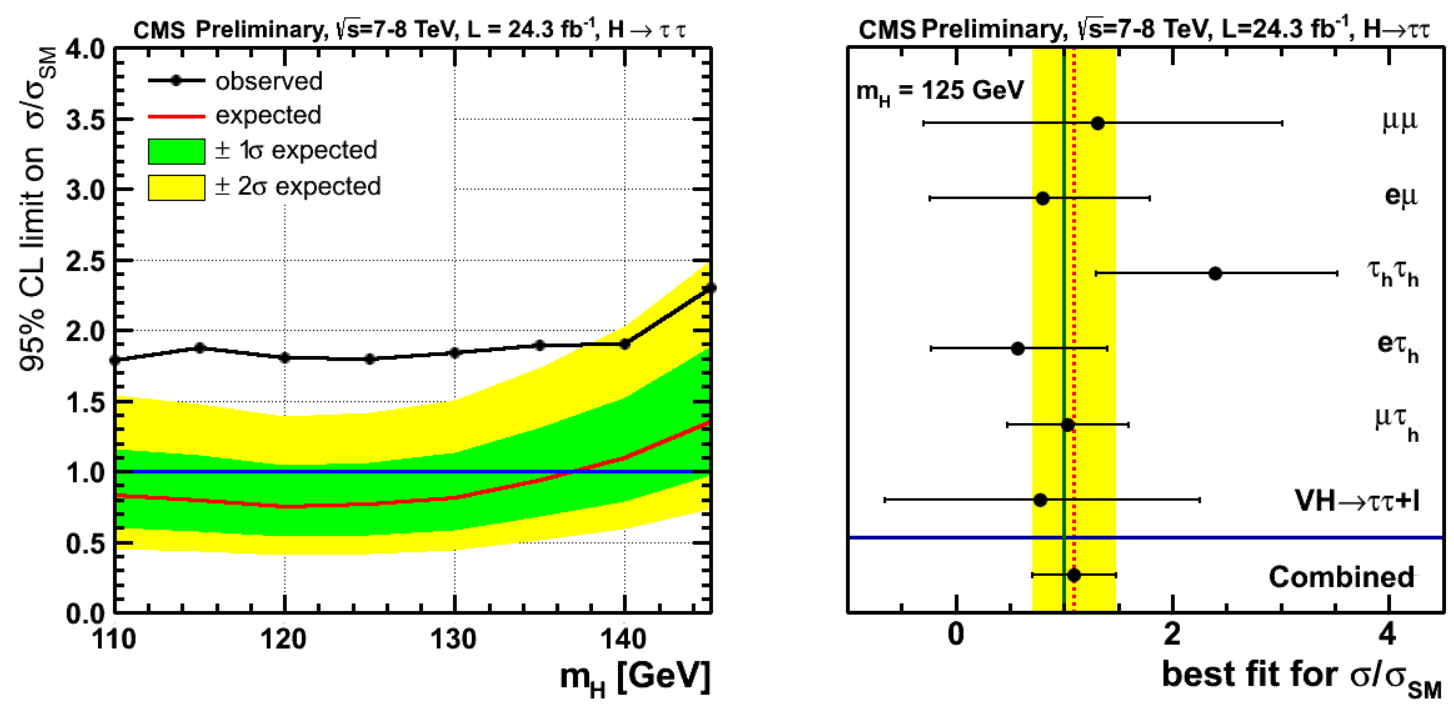

Figure 1: Left: The expected one- and two-standard-deviation ranges and observed 95\% CL upper limits on cross section normalized to the SM expectation as a function of the Higgs boson mass $\mathrm{m}_{\mathrm{H}}$. Right: Best-fit signal strength values, $\sigma / \sigma_{H}$, for independent channels. The green vertical line indicates the SM Higgs expectation.

azimuthal angle $(\phi)$, and are reconstructed as several photons. To increase the acceptance for such converted photons, the neutral pions are identified by clustering the reconstructed photons in narrow strips along the azimuthal $(\phi)$ direction. The $\tau_{h}$ isolation parameter $R_{\text {Iso }}^{\tau}$ is obtained from a multivariate discriminator, taking as input a set of transverse momentum sums $S_{j}=\sum_{i} p_{\mathrm{T}, i, j}$, where $p_{\mathrm{T}, i, j}$ is the transverse momentum of a particle $i$ in a ring $j$ centred on the $\tau_{h}$ candidate direction and defined in $(\eta, \phi)$ space. Five equal-width rings are used up to a distance $\Delta R=\sqrt{\Delta \eta^{2}+\Delta \phi^{2}}=0.5$ from the $\tau_{h}$ candidate, where $\Delta \eta$ and $\Delta \phi$ are the pseudorapidity and azimuthal angle differences, respectively, between the particle and the $\tau_{h}$ candidate direction. The effect of pileup on the isolation parameter is reduced mainly by discarding from $S_{j}$ calculation the charged hadrons with a track originating from pileup vertex. The efficiency achieved using the multivariate isolation is $65 \%$ over generated tau $\mathrm{p}_{\mathrm{T}}$ with a fake rate of jets mis-identified as hadronic taus around $2 \%$.

The $\mathrm{H} \rightarrow \tau \tau$ search [5] is performed using the final state signatures $\mu \tau_{h}$, e $\tau_{h}, \mathrm{e} \mu, \mu \mu$ and $\tau_{h} \tau_{h}$ where electrons and muons arise from leptonic $\tau$-decays and $\tau_{h}$ denotes hadronic tau decay. The di-tau mass reconstruction is done via a likelihood based technique based on tau decay kinematics and compatibility of reconstructed missing transverse energy with neutrino hypothesis. Each of these categories is further divided into three exclusive sub-categories according to the associated jets in the event. The full set of selected events is split into mutually exclusive categories based on the jet multiplicity, and on the transverse momentum of the reconstructed $\tau$-decay products. The selections are optimized to maximize the sensitivity of the analysis to the presence of a SM Higgs boson with a mass between $\mathrm{m}_{\mathrm{H}}=110$ and $145 \mathrm{GeV}$. The 0 -jet category is only used to constrain background normalization, identification efficiencies and energy scales. The 1-jet category exploits the production of a Higgs boson recoiling against a jet suppressing the background to significant extent. Events with high- $\mathrm{p}_{\mathrm{T}}$ Higgs bosons typically have much larger missing $\mathrm{E}_{\mathrm{T}}$ and benefit from 


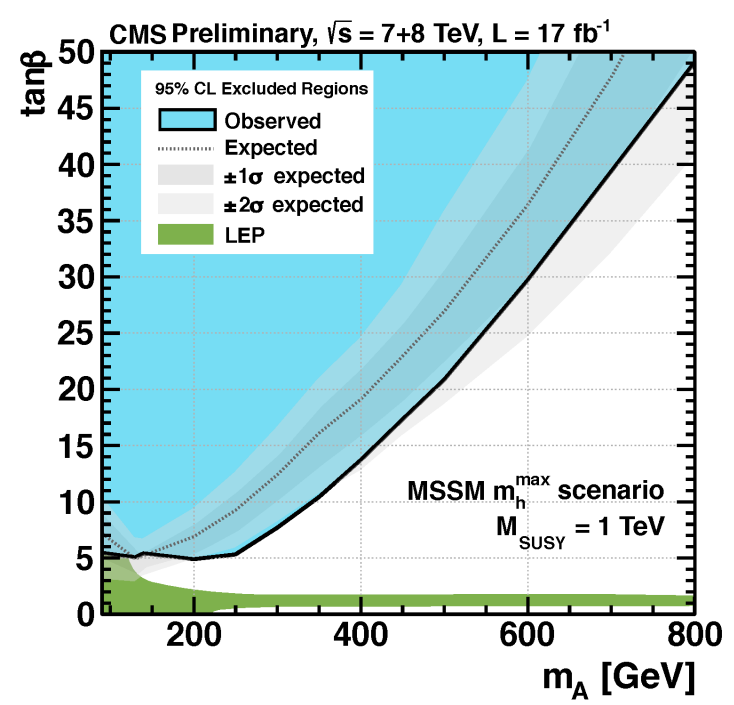

Figure 2: Exclusion in the MSSM parameter space on $\mathrm{M}_{\mathrm{A}}-\tan \beta$ plane for tau pair decay mode.

an improved resolution on this quantity, and thus on $m_{\tau \tau}$. The 2-jet category is required to tag the vector-boson fusion Higgs boson production process with the topology of two jets widely separated in pseudorapidity, which gives a high signal over background ratio.

In each of these categories a search is performed for a broad excess in the reconstructed di-tau invariant mass distribution. The largest source of irreducible background is $Z \rightarrow \tau \tau$ which is estimated using an observed sample of $Z \rightarrow \mu \mu$ events in data, where the reconstructed muons are then replaced by simulated tau leptons, a procedure called "embedding". The reducible backgrounds $(\mathrm{W}+\mathrm{jets}$, multijet production, $\mathrm{Z} \rightarrow \ell \ell$ ) are evaluated from control samples in data. The QCD multijet background is evaluated from same-sign data using the ratio of opposite-sign (OS) to same-sign (SS) data and relaxed lepton isolation after an estimate of $\mathrm{W}+$ jets background using control sample in data of $\mathrm{W}$ boson enriched region and extrapolating it to the signal region. The Z+jets background is evaluated using the rate of jets/leptons faking taus and OS/SS ratio with the shape from simulation. The top pair produced events and diboson contribution is estimated using simulation. Combining all categories, an excess of events is observed in the tau-pair invariant mass spectrum. For a SM Higgs boson in the mass range of 110 to $145 \mathrm{GeV}$, upper limits at $95 \%$ confidence level (CL) on the production cross section times the branching ratio to tau pairs are determined as seen in Figure 1 (left). A broad excess is observed over a range of the Higgs boson mass with an observed significance of $2.85 \sigma$ at $125 \mathrm{GeV}$. A signal injection test of $125 \mathrm{GeV}$ Higgs boson confirms that the excess observed is indeed consistent with the new boson discovered in the bosonic decay channels. The signal strength is also illustrated in Fig.1 (right) for different channels considered in the analysis and shows consistency across all channels. This result thus provides a strong affirmation of the Higgs boson coupling to fermions and a first indication of the Higgs coupling to leptons.

The production of neutral MSSM Higgs bosons proceed via gluon fusion through bottom / sbottom or top / stop loops, $b \bar{b}$ annihilation and production in association with a bottom quark. For the neutral MSSM Higgs bosons search, the data sample is divided into b-tag and non b-tag categories to enhance the sensitivity of the b-quark coupling to Higgs boson. 95\% CL upper bounds 
on the Higgs production cross-section times the branching fraction to tau pairs is obtained over the pseudoscalar Higgs boson mass $\left(\mathrm{M}_{\mathrm{A}}\right)$ and these limits are then interpreted in the MSSM parameter space of $\mathrm{M}_{\mathrm{A}}$ and $\tan \beta$, the ratio of the vacuum expectation values of the two Higgs doublets. The results obtained with almost $17 \mathrm{fb}^{-1}$ of 7 and $8 \mathrm{TeV}$ data set significantly constrains the MSSM parameter space, now reaching as low as $\tan \beta=4$ at $\mathrm{M}_{\mathrm{A}}=200 \mathrm{GeV}$ [6].

\section{3. $\mathbf{H} \rightarrow b \bar{b}$ decay mode}

The identification of bottom quarks is achieved by the Combined Secondary Vertex (CSV) b-jet tagging algorithm [4] that exploits all known variables, which can distinguish b-jets from non b-jets. Its goal is to provide optimal b-tag performance, by combining information about impact parameter significance, the secondary vertex and jet kinematics. An efficiency of $70 \%$ is achieved from top pair and muon+jets sample for a fake rate of $2 \%$. With the b-quarks identified using CSV algorithm, a search for the SM Higgs boson decaying to a pair of bottom quarks [7] when produced in association with weak vector bosons $(\mathrm{V})$ is reported for the following modes: $\mathrm{W}(\mu \nu) \mathrm{H}$, $\mathrm{W}(\mathrm{e} v) \mathrm{H}, \mathrm{Z}(\mu \mu) \mathrm{H}, \mathrm{Z}(\mathrm{ee}) \mathrm{H}$ and $\mathrm{Z}(v v) \mathrm{H}$. The search is performed with $5.0 \mathrm{fb}^{-1}$ of $7 \mathrm{TeV}$ pp data sample from 2011, and up to $12.1 \mathrm{fb}^{-1}$ of $8 \mathrm{TeV}$ data during 2012. The background processes to $\mathrm{VH}$ production are vector boson + jets, $t \bar{t}$, single top, dibosons (VV) and QCD multijet production. The event selection for a Boosted Decision Tree (BDT) analysis is based first on the kinematic reconstruction of the vector bosons and the Higgs boson decay into two b-tagged jets. Backgrounds are then substantially reduced by requiring a significant boost in the $\mathrm{p}_{\mathrm{T}}$ of the vector boson and the Higgs boson. Appropriate control regions that are orthogonal to the signal region are identified in data and used to adjust the Monte Carlo simulation normalization for the most important background processes: $\mathrm{W}+\mathrm{jets}$ and $\mathrm{Z}+\mathrm{jets}$ (with light- and heavy-flavor jets), and top production.

A b-jet energy regression is trained to improve the reconstructed mass resolution, using secondary vertex and jet properties, missing transverse energy direction and soft lepton information inside the jet, which improves the overall analysis sensitivity by 15 to $20 \%$. The dijet mass resolution is close to $10 \%$. In each of the channels, a BDT is used to increase the discrimination against background (mainly vector boson plus jets, dibosons and top), which takes as input, jets and vector bosons kinematics and b-tagging discriminant information. A shape analysis of the BDT output is performed in each channel which leads to an improvement of $10 \%$ with respect to the case where only a sequential criterion on the BDT output is used.

Upper limits, at $95 \%$ confidence level, on the VH production cross section times the $\mathrm{H} \rightarrow b \bar{b}$ branching ratio, with respect to the expectations for a SM Higgs boson, are derived in the mass range 110 to $135 \mathrm{GeV}$. In this range, the observed upper limits vary from 1.0 to 4.7 times the standard model prediction; the corresponding expected limits vary from 0.9 to 1.9. At a Higgs boson mass of 125 $\mathrm{GeV}$ the observed limit is 2.5 and the expected limit is 1.2. The excess of observed events is 2.2 standard deviations from expected background only contribution, and is consistent with the SM prediction for Higgs boson production.

A search for neutral MSSM Higgs bosons decaying to a pair of b quarks in association with at least one additional $\mathrm{b}$ quark is presented [8]. The data corresponds to $2.7-4.8 \mathrm{fb}^{-1}$ collected by the CMS detector in pp collisions at a centre-of-mass energy of $7 \mathrm{TeV}$. This search is particularly sensitive to Higgs bosons in MSSM scenarios with large values of $\tan \beta$. We study multijet final 

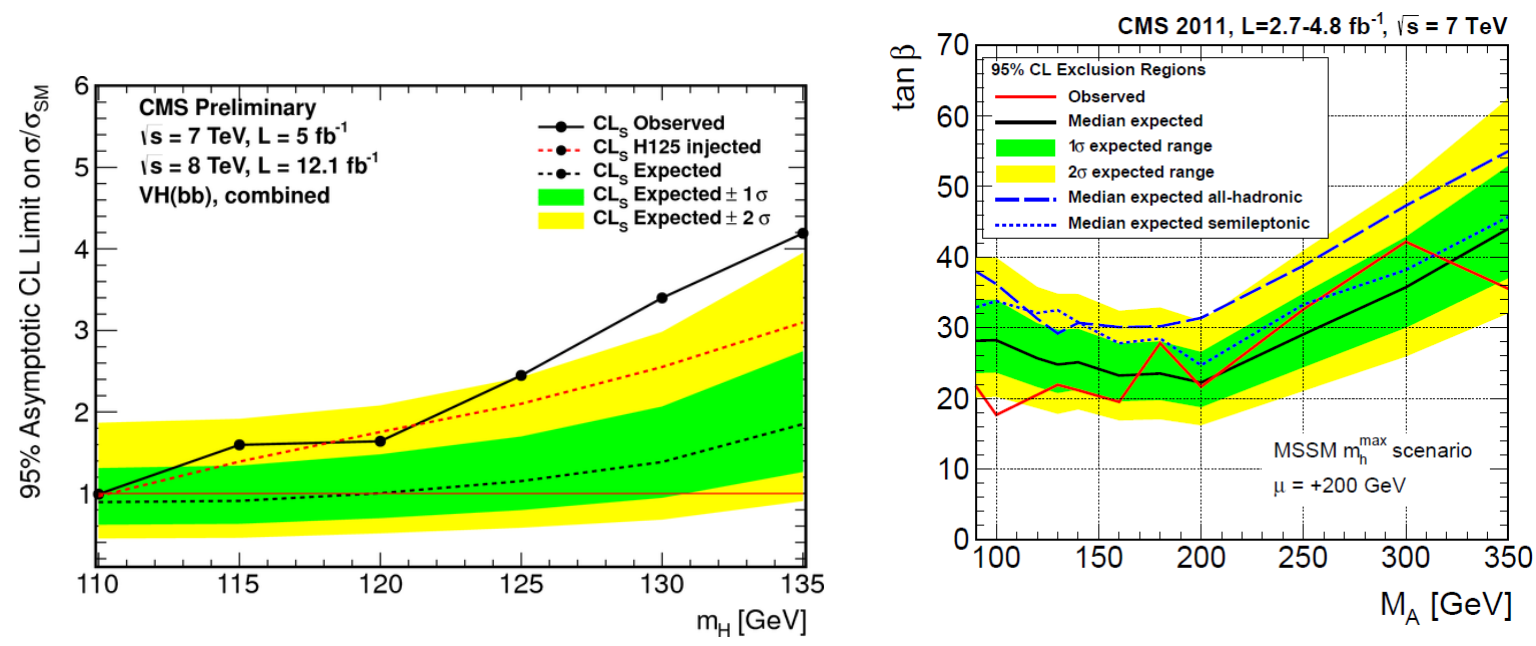

Figure 3: Left: The expected one- and two-standard-deviation ranges and observed 95\% CL upper limits on cross section normalized to the SM expectation as a function of the Higgs boson mass $\mathrm{m}_{\mathrm{H}}$. Right: Exclusion in the MSSM parameter space on $\mathrm{M}_{\mathrm{A}}$-tan $\beta$ plane for bottom pair decay mode.

states with three b-tagged jets, one of which may include a non-isolated muon. The heavy flavor multijet background is derived from data. No excess has been observed and limits have been placed in the MSSM parameter space within the $\mathrm{m}_{h}^{\max }$ scenario reaching $\tan \beta=18$ at $\mathrm{M}_{\mathrm{A}}=100 \mathrm{GeV}$.

\section{Conclusion}

Results for the SM and MSSM Higgs boson search decaying to a pair of tau leptons and bottom quarks have been presented. CMS has observed a broad excess for the SM Higgs search in the tau pair decay mode in the low mass range with a significance of $2.85 \sigma$ at $\mathrm{m}_{\mathrm{H}}=125 \mathrm{GeV}$ and this is consistent with the new boson discovered in the bosonic decay channels. The result is a strong affirmation of the Higgs coupling to fermions and a first indication of the Higgs coupling to leptons. The excess in $b \bar{b}$ channel has a significance of $2.2 \sigma$ and is consistent with SM Higgs boson production. The search for MSSM Higgs bosons has significantly constrained limits in the $\left(\mathrm{M}_{\mathrm{A}}-\tan \beta\right)$ plane in the $\tau \tau$ and $b \bar{b}$ decay modes.

\section{References}

[1] CMS Collaboration, CMS Physics Analysis Summary CMS-HIG-13-005; CMS-HIG-12-045.

[2] CMS Collaboration, JINST 0803 (2008) S08004.

[3] CMS Collaboration, JINST 7 (2012) P01001.

[4] CMS Collaboration, JINST 8 (2013) P04013.

[5] CMS Collaboration, CMS Physics Analysis Summary CMS-HIG-13-004 and references therein.

[6] CMS Collaboration, CMS Physics Analysis Summary CMS-HIG-12-050 and references therein.

[7] CMS Collaboration, CMS Physics Analysis Summary CMS-HIG-12-044 and references therein.

[8] CMS Collaboration, arXiv: 1302.2892 [hep-ex] and references therein. 